\title{
Temporary cooling of quasiparticles and delay in voltage response of superconducting bridges after abruptly switching on the supercritical current
}

\author{
D. Yu. Vodolazov ${ }^{1,2, *}$ and F. M. Peeters ${ }^{3}$ \\ ${ }^{1}$ Institute for Physics of Microstructure, Russian Academy of Sciences, 603950 Nizhny Novgorod, GSP-105, Russia \\ ${ }^{2}$ Lobachevsky State University of Nizhny Novgorod, 23 Gagarin Avenue, 603950 Nizhny Novgorod, Russia \\ ${ }^{3}$ Departement Fysica, Universiteit Antwerpen (CGB), Groenenborgerlaan 171, B-2020 Antwerpen, Belgium
}

(Received 13 May 2014; revised manuscript received 26 August 2014; published 10 September 2014)

\begin{abstract}
We revisit the problem of the dynamic response of a superconducting bridge after abruptly switching on the supercritical current. In contrast to previous theoretical works we take into account spatial gradients and use both the local temperature approach and the kinetic equation for the distribution function of quasiparticles. We find that the temperature dependence of the finite delay time $t_{d}$ in the voltage response is model dependent and relatively large $t_{d}$ is connected with temporary cooling of quasiparticles during decay of superconducting order parameter $|\Delta|$ in time. It turns out that the presence of even small inhomogeneities in the bridge or finite length of the homogenous bridge favors a local suppression of $|\Delta|$ during the dynamic response. It results in a decrease of the delay time, in comparison with the spatially uniform model, due to the diffusion of nonequilibrium quasiparticles from the region with locally suppressed $|\Delta|$. In the case when the current density is maximal near the edge of a not very wide bridge the delay time is mainly connected with the time needed for the nucleation (entrance) of the first vortex and $t_{d}$ could be tuned by a weak external magnetic field. We also find that a short alternating current pulse (sinusoidlike) with zero time average may result in a nonzero time-averaged voltage response where its sign depends on the phase of the ac current.
\end{abstract}

DOI: 10.1103/PhysRevB.90.094504

PACS number(s): 74.25.F-, 74.40.Gh

\section{INTRODUCTION}

In 1979 Pals and Wolter [1] observed a long time delay (about hundreds of nanoseconds) in the appearance of the voltage response after the instant (on a time scale of $1 \mathrm{~ns}$ ) application of the supercritical current to an Al superconducting film. This work initiated a large number of studies (both experimental and theoretical) which aimed to clarify the physical origin of this effect [2-8]. The main conclusion was that during transition the superconducting order parameter $\Delta=|\Delta| \exp (i \phi)$ decays in time and it provides some kind of temporary "cooling" of quasiparticles [2,9]. Because the critical current in superconductors increases, when the temperature decreases, this effective cooling shifts the applied current $I>I_{c}(T)$ closer to the nonequilibrium $I_{c}$ (corresponding to lower "temperature") and it slows down the destruction of superconductivity. It was experimentally observed that the time delay $t_{d}$ decreases fast with increasing amplitude of the current and in some experiments a strong dependence of $t_{d}$ on temperature was found [4-7], while in other works $t_{d}$ practically did not depend on $T[1,8]$.

In the majority of previous theoretical studies on this subject authors assumed that superconductivity decays uniformly in space [1-3] which considerably simplified the calculations. Besides it was assumed that the inelastic electron-electron relaxation time $\tau_{e-e}$ is much larger than the inelastic electronphonon relaxation time $\tau_{e-p h}$, and the escape time of the nonequilibrium phonons $\tau_{e s c}$ to the substrate is smaller than $\tau_{e-p h}$. These assumptions lead to the nonthermal nonequilibrium quasiparticle distribution function $f(\epsilon)$ (i.e., it cannot be described by the Fermi-Dirac distribution function with an

\footnotetext{
*vodolazov@ipmras.ru
}

effective temperature and chemical potential) and the phonons being in equilibrium.

In the present work we extend the model of Tinkham [2] to the spatially nonuniform case (to study the bridges with finite length and with small inhomogeneities) and to the case when the deviation from equilibrium could be described in terms of a local temperature (which is valid when $\tau_{e-e} \ll \tau_{e-p h}$-the so-called quasiequilibrium limit $[10,11])$. We find that the last model predicts the temperature-independent delay time, which was earlier observed in some experiments [1,8], and which was not explained by previous theoretical works. We show that the presence of the intrinsic defects in the bridge and/or contacts with the wider/thicker superconductors decreases the time delay, because in this case the order parameter decays faster in some "weak" places and cooling of quasiparticles becomes nonuniform in space.

Another important question which was not studied before is how the nonuniform current density distribution in the film/wire influences the time delay. Nonuniformity may come from the Meissner (screening) effect which is important in wide films/bridges with width $w>\lambda^{2} / d$ ( $\lambda$ is the London penetration depth, $d$ is the thickness of the film/bridge). In narrow films/bridges $\left(w \ll \lambda^{2} / d\right)$ spatially nonuniform current distribution may arise due to the current crowding effect near edge/surface irregularities [12-14] or due to specific geometry [15]. We demonstrate, that for not very wide bridges and currents close to $I_{c}$, the delay in the voltage response is mainly connected with the entrance of the first vortex and qualitatively resembles the time delay of quasi-onedimensional (1D) bridges. We also offer to use the weak magnetic field for tuning the time delay via change of the current distribution in the film.

The third problem which we investigate is the response of the superconducting bridge on a short alternating current pulse. 
Our interest in this problem arises from a recent experiment where the nonzero time-averaged voltage response of a wide yttrium barium copper oxide (YBCO) superconducting bridge to a short pulse of synchrotron radiation was observed [16]. This experiment demonstrates the possibility to study the resistive response of the superconducting bridge on a very short time scale (approximately several picoseconds) when the superconductor cannot be substantially heated and which is hard to realize by other methods. Using our theoretical models we also find the nonzero time-averaged voltage response on the zero time-averaged ac current pulse which we explain by the long delay time in the destruction of superconductivity in the bridge by the supercritical current. Moreover we predict that the time-averaged voltage changes the sign with the shift of the phase of ac current pulse by $\pi$.

The structure of our paper is as follows. In Sec. II we present our theoretical model that we use to study the dynamic response of "dirty" superconductors near its critical temperature $T_{c}$. In Sec. III we present the results of our calculations for quasi-1D bridges in the nonthermal (Sec. III A) and quasiequilibrium (Sec. III B) cases. Subsequently, in Sec. IV we present results for wide $[w \gg \xi(T)]$ superconducting bridges placed in a weak magnetic field (which creates a nonuniform current distribution) and in Sec. V we study the dynamic response of the superconducting bridge on a sinusoidal-like current pulse. In Sec. VI we discuss the applicability of our results to different physical situations.

\section{MODEL}

To model the dynamical response of the "dirty" superconductor near $T_{c}$ we use the simplified set of equations which was derived in Refs. [17-21]. First of all near $T_{c}$ one may neglect the coupling between odd $f_{L}(\epsilon)$ and even $f_{T}(\epsilon)$ energy parts of the quasiparticle distribution function $f(\epsilon)=(1-$ $\left.f_{L}-f_{T}\right) / 2$ [nonequilibrium $f_{L}(\epsilon)$ describes effects connected with heating/cooling of quasiparticles, while finite $f_{T}(\epsilon) \neq$ 0 describes effects connected with charge imbalance and normal current in the superconductor]. Secondly, instead of solving the kinetic equation for $f_{T}(\epsilon)$ we use the following expression:

$$
f_{T}=-e \varphi \frac{\partial f_{L}^{0}}{\partial \epsilon}
$$

where $\varphi$ is the electrical potential, $f_{L}^{0}=\tanh \left(\epsilon / 2 k_{B} T\right)$, and one assumes a small deviation from equilibrium $\delta f_{L}=f_{L}-$ $f_{L}^{0} \ll f_{L}^{0}$. Equation (1) satisfies the kinetic equation for $f_{T}$ [see, for example, Eq. (7) in Ref. [19]] when one neglects the second spatial derivative of $f_{T}$ and $e \varphi+\hbar \partial \phi / \partial t=0$. Both these conditions are approximately valid during almost all of the transition period when there is a small gradient of the superconducting current and, hence, the small gradient of the normal current. We also do not expect large changes in our results (at temperatures not very close to $T_{c}$ ) at the last stage of the transition period and during formation of the phase slip line because Eq. (1) gives practically the same conversion length of the normal current to the superconducting one [of about coherence length $\xi(T)]$ as one expects at $T \lesssim 0.9 T_{c}$ [22].
With these simplifications the equations for $f_{L}$ and $\Delta$ have the following form:

$$
\begin{gathered}
N_{1} \frac{\partial f_{L}}{\partial t}=D \nabla\left[\left(N_{1}^{2}-R_{2}^{2}\right) \nabla f_{L}\right]-\frac{N_{1}}{\tau_{\text {in }}}\left(f_{L}-f_{L}^{0}\right) \\
-R_{2} \frac{\partial f_{L}^{0}}{\partial \epsilon} \frac{\partial|\Delta|}{\partial t}, \\
\frac{\pi \hbar}{8 k_{B} T_{c}}\left(\frac{\partial}{\partial t}+\frac{2 i e \varphi}{\hbar}\right) \Delta=\xi_{G L}^{2}\left(\nabla-i \frac{2 e}{\hbar c} A\right)^{2} \Delta \\
+\left(1-\frac{T}{T_{c}}+\Phi_{1}-\frac{|\Delta|^{2}}{\Delta_{G L}^{2}}\right) \Delta,
\end{gathered}
$$

where $\xi_{G L}^{2}=\pi \hbar D / 8 k_{B} T_{c}$ and $\Delta_{G L}^{2}=8 \pi^{2}\left(k_{B} T_{c}\right)^{2} / 7 \zeta(3)$ are the zero temperature Ginzburg-Landau coherence length and the order parameter, correspondingly, $A$ is the vector potential, and $\Phi_{1}=\int_{0}^{\infty} R_{2} \delta f_{L} d \epsilon /|\Delta|$. From Eq. (3) it follows that for the uniform case and in equilibrium $|\Delta|=\Delta_{e q}=\Delta_{G L}(1-$ $\left.T / T_{c}\right)^{1 / 2}$.

To find the solution of Eq. (2) one should use the Usadel equation for the normal $\alpha(\epsilon)=\cos \Theta=N_{1}(\epsilon)+i R_{1}(\epsilon)$ and anomalous $\beta_{1}=\beta e^{i \phi}, \beta_{2}=\beta e^{-i \phi}\left[\beta(\epsilon)=\sin \Theta=N_{2}(\epsilon)+\right.$ $i R_{2}(\epsilon)$ ] Green functions

$$
\left[\left(2 i \epsilon-\frac{\hbar}{\tau_{\text {in }}}\right)-\frac{D}{\hbar} q_{s}^{2} \cos \Theta\right] \sin \Theta+2|\Delta| \cos \Theta=0,
$$

where $q_{s}=m v_{s}=(\nabla \phi-2 e A / \hbar c)$ is the superfluid momentum. In Eq. (4) we skip the term with the spatial derivative. We checked out that its presence weakly affects the time delay at $T \geqslant 0.9 T_{c}$. In contrast, we find that the term proportional to $q_{s}^{2}$ leads to a considerable decrease of the time delay.

Within the same approximation the current density in the superconductor may be written as

$$
\begin{aligned}
j & =\frac{\sigma_{n}}{e} \frac{\pi|\Delta|^{2} q_{s}}{4 k_{B} T_{c}}+\frac{\sigma_{n}}{e} \int_{0}^{\infty} j_{\epsilon} \delta f_{L} d \epsilon-\sigma_{n} \nabla \varphi \\
& =j_{s}+\delta j_{s}+j_{n},
\end{aligned}
$$

where the first term on the right-hand side (RHS) is the superconducting current density; $j_{n}=-\sigma_{n} \nabla \varphi\left(\sigma_{n}=2 e^{2} D N_{0}\right)$ is the normal current density $\left(N_{0}\right.$ is the one spin density of states at the Fermi level). In Eq. (5) we keep the nonequilibrium contribution to the supercurrent $\left(\delta j_{s}\right)$ due to $\delta f_{L} \neq 0$. In Ref. [3] it was argued that the presence of this term increases $t_{d}$ when $I \gg I_{c}$.

When the escape time of phonons to the substrate is shorter than the inelastic electron-phonon relaxation time and at the same time the electron-electron scattering is weaker than the electron-phonon one, then the relaxation time in Eq. (2) $\tau_{\text {in }}=\tau_{e-p h}$ and the quasiparticle distribution function is not a thermal one. In this case Eqs. (2)-(4) and the current continuity equation $\operatorname{div} j=0$ are the equations that govern the dynamic response of the superconducting bridge to a supercritical current.

In the opposite limit the quasiparticles are thermalized due to electron-electron inelastic scattering and $f(\epsilon)$ can be described by the Fermi-Dirac distribution function with a local temperature and chemical potentialthe so-called 
quasiequilibrium approach [10,11] [in this limit one has to take into account the electron-electron collision integral in Eq. (2), which is omitted there]. From Eqs. (2) and (4) one may obtain (see Appendix) the heat conductance equation for the local temperature of the quasiparticles $T_{\text {loc }}=T+\delta T_{\text {loc }}$,

$$
C_{v} \frac{\partial \delta T_{\mathrm{loc}}}{\partial t}=\kappa \nabla^{2} \delta T_{\mathrm{loc}}+N_{0} \frac{T}{T_{c}} \frac{\partial|\Delta|^{2}}{\partial t}-C_{v} \frac{\delta T_{\mathrm{loc}}}{\tau_{\mathrm{in}}},
$$

where $C_{v}=2 \pi^{2} k_{B}^{2} N_{0} T / 3$ is the electron heat capacity and $\kappa=2 \pi^{2} k_{B}^{2} D N_{0} T / 3$ is the electron heat conductivity in the normal state. In this limit $\tau_{\text {in }}=\tau_{e-p h}$ or $\tau_{e s c}$, whatever is larger. In Eq. (6) we neglect the heating effects due to Joule dissipation which is valid for our problem near $T_{c}$ (for discussion see Appendix).

The time-dependent equation for $\Delta$ in this limiting case resembles the ordinary time-dependent Ginzburg-Landau equation with time- and coordinate-dependent local temperature:

$$
\begin{aligned}
& \frac{\pi \hbar}{8 k_{B} T_{c}}\left(\frac{\partial}{\partial t}+2 i e \varphi\right) \Delta \\
& \quad=\xi_{G L}^{2}\left(\nabla-i \frac{2 e}{\hbar c} A\right)^{2} \Delta+\left(1-\frac{T_{\mathrm{loc}}}{T_{c}}-\frac{|\Delta|^{2}}{\Delta_{G L}^{2}}\right) \Delta .
\end{aligned}
$$

[in this form Eq. (7) was derived earlier in Ref. [23]].

Equations (6) and (7) and $\operatorname{div} j=0$ are the basic equations that govern the transient response of the superconducting bridge in the quasiequilibrium approach.

We define the time delay $t_{d}$ as the time needed for suppression of $|\Delta|$ from its equilibrium value $|\Delta|=\Delta_{e q}$ (at $t \leqslant 0$, when the current is equal to zero) to $|\Delta|=0$ anywhere in the superconductor. In the analytic models there are "forbidden" values of $|\Delta|$ (see Sec. III) and the time delay is defined as the time needed for change of $|\Delta|$ from some maximal up to a minimal "available" value.

For the 1D case we assume that the superconducting bridge of finite length $L$ is attached to massive superconducting electrodes which are in equilibrium (physically it corresponds to the variable thickness bridge). It imposes the following boundary conditions: $\Delta(x=0, L)=\Delta_{e q} \exp [i \varphi(x=0, L) t]$, $T_{\text {loc }}(x=0, L)=T, \varphi(0)=0, \varphi(L)=V$, and the voltage $V$ can be found from integrating Eq. (5) over the length of the bridge. For $f_{L}$ more complicated boundary conditions are used: $f_{L}(x=0, L)=f_{L}^{0}$ when $\epsilon>\Delta_{e q}$ and $\partial f_{L} / \partial x(x=$ $0, L)=0$ for smaller energies.

In the two-dimensional case we assume that the superconducting bridge of finite length $L$ and finite width $w$ is attached to massive normal electrodes being in equilibrium. Normal electrodes considerably simplify our calculation in the current constant regime, which could be easily implemented via boundary conditions for the electrostatic potential $-\sigma_{n} \partial \varphi / \partial x(x=0, L)=j$. The rest of the boundary conditions are as follows: in the longitudinal $(x)$ direction $\Delta(x=0, L)=$ $0, T_{\mathrm{loc}}(x=0, L)=T$, and $f_{L}(x=0, L)=f_{L}^{0}$, and in the transverse $(y)$ direction we use ordinary superconductorisolator boundary conditions. To diminish the influence of nonequilibrium effects from normal-superconductor (NS) boundaries we locally increase $T_{c}$ near the ends of the bridge (on the distance $5 \xi_{0}$ from each end) by $20 \%$.

In our calculations we use the following natural variables as the units of the corresponding quantities: $t_{0}=\hbar / \Delta_{0}, \Delta_{0}=$ $1.76 k_{B} T_{c}, \quad \xi_{0}=\sqrt{\hbar D / \Delta_{0}}, q_{s}^{0}=\hbar c / 2 e \xi_{0}, \quad j_{0}=\Delta_{0} \sigma_{n} /\left(\xi_{0} e\right)$, and $\varphi_{0}=\Delta_{0} / e$. We use $\tau_{\text {in }} / t_{0}=8-1000$ which are typical values for many superconductors (for example in $\mathrm{MgB}_{2}$ $\tau_{e-p h} / t_{0} \simeq 20$, in $\mathrm{Nb} \tau_{e-p h} / t_{0} \simeq 100$, in Sn $\tau_{e-p h} / t_{0} \simeq 200$, and in $\mathrm{Al} \tau_{e-p h} / t_{0} \simeq 1000$ ).

In simulations the current increases linearly (from $t=0$ ) during the time interval $\delta t=5 t_{0}$. Such a procedure provides a better numerical stability of our calculations in comparison with an instant application of the current. Time delay depends weakly on the specific choice of $\delta t$ while $t_{d} \gg \delta t$.

\section{TRANSIENT RESPONSE IN THE 1D CASE}

\section{A. Nonthermal model}

For simplicity we first neglect the nonequilibrium contribution $\delta j_{s}$ to the supercurrent. The effect of finite $\delta j_{s}$ on $t_{d}$ will be discussed at the end of this section.

In the spatially uniform case one may find from Eqs. (2) and (3) the equation for the dynamics of the dimensionless magnitude of the order parameter $f=|\Delta| / \Delta_{e q}$,

$$
\begin{aligned}
& \tau_{G L} \frac{\partial f}{\partial t}+\operatorname{afY}(f, T) \int_{0}^{t} \frac{\partial f}{\partial t^{\prime}} e^{-\left(t-t^{\prime}\right) / \tau_{\text {in }}} d t^{\prime} \\
& \quad=f\left(1-f^{2}-\frac{\tilde{I}_{s}^{2}}{f^{4}}\right),
\end{aligned}
$$

where $a=\pi \Delta_{G L} / 4 k_{B} T\left(1-T / T_{c}\right)^{1 / 2} \simeq 2.4 /\left(1-T / T_{c}\right)^{1 / 2}$, $\tau_{G L}=\pi \hbar / 8 k_{B}\left(T-T_{c}\right)$,

$$
Y(f, T)=\frac{2}{\pi} \int_{1}^{\infty} \frac{d \epsilon}{\epsilon\left(\epsilon^{2}-1\right)^{1 / 2} \cosh \left(\epsilon f \Delta_{e q} / 2 k_{B} T\right)},
$$

and $\tilde{I}_{s}^{2}=4 / 27\left(I_{s} / I_{\mathrm{dep}}\right)^{2} \quad\left(I_{\mathrm{dep}}=j_{\mathrm{dep}} w d\right.$ is the GinzburgLandau depairing current, $I_{s}=j_{s} w d$ and we use the initial condition $f_{L}=f_{L}^{0}$ at $t=0$ when the current is turned on). In the case when $\Delta_{e q} / 2 k_{B} T \leqslant 1 / 2$ one has $Y(f, T) \simeq$ $1-b f\left[b=\Delta_{e q} / 2 k_{B} T \simeq 1.52\left(1-T / T_{c}\right)^{1 / 2}\right]$. Note that in Refs. [2,3] $Y(f)=1$, which is valid when $b \ll 1$. But $b \simeq$ 0.48 already at $T=0.9 T_{c}$ and the term $-b f$ in $Y(f)$ should be taken into account.

In Eq. (8) one may neglect the first term on the left-hand side (LHS) in comparison with the second term when $\tau_{G L} \ll$ $\tau_{\text {in }} /\left(1-T / T_{c}\right)^{1 / 2}$ [except the very beginning of the transition period when the time integral in Eq. (8) is small]. With this simplification from Eq. (8) one may find the differential equation for $f$

$$
\tau_{\text {in }} \frac{\partial f}{\partial t}=\frac{1-f^{2}-\tilde{I}_{s}^{2} / f^{4}}{a Y(f, T)+2 f-4 \tilde{I}_{s}^{2} / f^{4}+Y^{\prime}(f, T)\left(1-f^{2}-\tilde{I}_{s}^{2} / f^{4}\right) / Y(f, T)} .
$$


In the limit $Y(f, T)=1$ Eq. (10) coincides with Eq. (64) of Ref. [2]. Equation (10) becomes invalid at $f=f_{\min }$ when the denominator goes to zero

$$
a Y\left(f_{\min }\right)+2 f_{\min }-4 \tilde{I}_{s}^{2} / f_{\min }^{4}+Y^{\prime}\left(f_{\min }\right)\left(1-f_{\min }^{2}-\tilde{I}_{s}^{2} / f_{\min }^{4}\right) / Y\left(f_{\min }\right)=0,
$$

and in the beginning of the transition period when $f$ changes on a time scale $\sim \tau_{G L}$ from 1 to $f_{\max }<1$. $f_{\max }$ could be found from the following equation:

$$
\tilde{I}_{s}^{2}=\left(1-f_{\max }\right) f_{\max }^{4}\left[a Y\left(f_{\max }\right)+1+f_{\max }\right]
$$

which results from Eq. (8) if one assumes a steplike (on a time scale $\tau_{G L} \ll \tau_{\text {in }}$ ) decrease of $f$ from 1 to $f_{\max }$ [Eq. (12) transits to Eq. (62) of Ref. [2] when $\left.Y\left(f_{\max }\right)=1\right]$.

From Eqs. (10)- (12) one can find the delay time

$$
\frac{t_{d}}{\tau_{\text {in }}}=\int_{f_{\max }}^{f_{\min }} \frac{a Y(f, T)+2 f-4 \tilde{I}^{2} / f^{4}+Y^{\prime}(f, T)\left(1-f^{2}-\tilde{I}^{2} / f^{4}\right) / Y(f, T)}{1-f^{2}-\tilde{I}^{2} / f^{4}} d f,
$$

where we replace the superconducting current $I_{s}$ by the full current $I$ because when $f$ changes from $f_{\max }$ to $f_{\min }$ the normal current in the wire is much smaller than $I$ and $I_{s} \simeq I$.

In the spatially nonuniform case we solve the set of Eqs. (2)-(5) numerically [in Eq. (5) we put $\delta j_{s}=0$ ] and we find that even for a homogenous bridge, $|\Delta|$ decays faster near the ends of the bridge. At first sight this result looks rather unexpected, because at the ends $|\Delta|$ is maximal due to the boundary conditions (which originate from the proximity with the massive superconducting leads being in equilibrium). But effective cooling of quasiparticles is weaker near the ends of the bridge, because nonequilibrium quasiparticles with energy $\epsilon>\Delta_{e q}$ can freely diffuse away from the bridge to the leads. As a result $|\Delta|$ decreases faster near the ends of the bridge.

We checked that a similar effect exists (see also Ref. [24]) even in the so-called local equilibrium limit [when $L_{\text {in }}=$ $\left.\left(D \tau_{\text {in }}\right)^{1 / 2} \ll \xi(T)[19,20]\right]$ and one can neglect the diffusion of quasiparticles. But in that case the spatial gradient of $|\Delta|$ along the bridge is considerably smaller and the effect appears only in some range of currents and $\tau_{\text {in }}$. This result shows that not only diffusion of nonequilibrium quasiparticles may provide this effect but also the gradient of $|\Delta|$ which appears near the ends of the variable thickness bridge when $I \neq 0$.

Real bridges are never homogenous. Variations of their physical (mean free path length and/or $T_{c}$ ) and geometrical (width or thickness) properties along the superconductor may exist. For example we find that even a $2 \%$ suppression of $T_{c}$ on a length scale of $\xi_{0}$ in the center of the bridge favors the local suppression of $|\Delta|$ in comparison with its suppression near the ends of the bridge (in the studied temperature interval $0.9-0.98 T_{c}$ ). We checked that the time delay $t_{d}$ varies with a change of local $T_{c}$ (see Fig. 1) but the functional dependence $t_{d}\left(I / I_{c}\right)$ stays practically the same when the suppression of $I_{c}$ due to defects is not strong $\left(I_{c} \simeq I_{\text {dep }}\right)$. Further we consider the superconducting bridge with a defect in the center where $T_{C}$ is locally suppressed by $2 \%$.

In Fig. 2 we present the time evolution of the order parameter in the center of the superconducting bridge with length $60 \xi_{0}$ at $T=0.9 T_{c}$ and two values of $\tau_{\text {in }}$. One can see that qualitatively the dynamics of $|\Delta|$ follows the predictions of the spatially uniform model. At the beginning of the transition there is a sudden drop in $|\Delta|$ (on a time scale $\sim \tau_{G L}$ ) and the value of this drop is close to the one predicted by Eq. (12) (note that it does not depend on $\tau_{\text {in }}$ ). Further decay of $|\Delta|$ strongly depends on $\tau_{\text {in }}$ (see Fig. 2) until it reaches some minimal value which is close to the one predicted by Eq. (11). After that $|\Delta|$ varies fast in time (again on the time scale $\sim \tau_{G L}$ ) and its dynamics weakly depends on $\tau_{\text {in }}$.

In Fig. 3 we present the dependence $t_{d}\left(I / I_{c}\right)$ for different values of $\tau_{\text {in }}$. If one compares this result with Eq. (13) (solid curve in the inset of Fig. 3) one can see that for the spatially nonuniform case the time delay is much shorter (when $\tau_{\text {in }} \gg$ $\tau_{G L}$ ). Besides there is no "universal" curve (see the inset in Fig. 3 for normalized variables) on which all curves drop as in the case of the spatially uniform model. We explain these deviations by the diffusion of nonequilibrium quasiparticles from the point where $|\Delta|$ decreases faster. Indeed, the diffusion decreases locally the cooling of quasiparticles and accelerates the decay of $|\Delta|$. This effect also results in a dependence of $t_{d}$

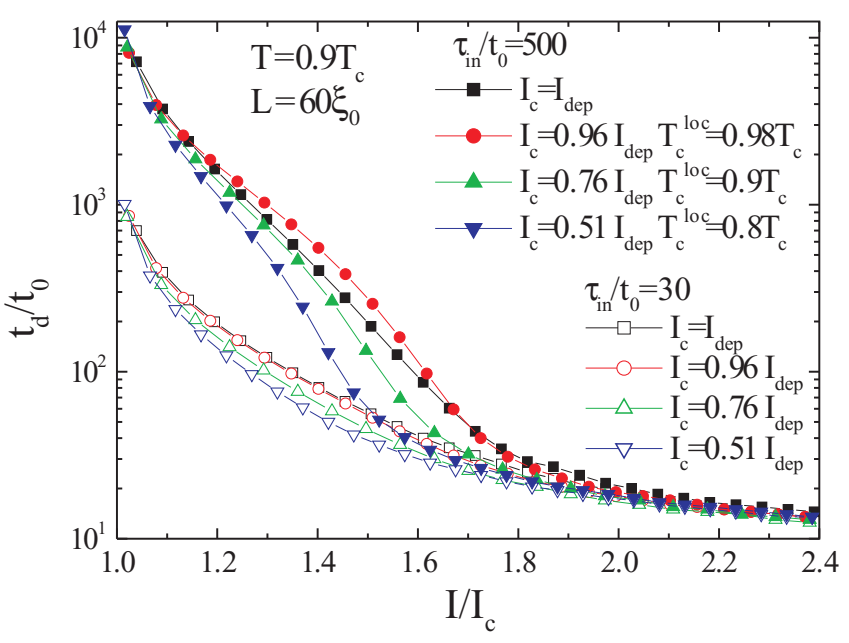

FIG. 1. (Color online) Dependence of the time delay $t_{d}$ on the normalized current $I / I_{c}$ in the superconducting bridge with locally suppressed $T_{c}^{\text {loc }}$ (in the area with size $\xi_{0}$ in the center of the bridge) and two values of $\tau_{\text {in }}$. Decrease of $t_{d}$ with decreasing $T_{c}^{\text {loc }}$ is explained by the locally smaller value of $|\Delta|$ in equilibrium and it takes less time to suppress it to zero. In the case of a defect-free bridge $\left(I_{c}=I_{\mathrm{dep}}\right)$ the time delay is smaller than in the bridge with a weak defect (at the same value of normalized current $\left.I / I_{c}\right)$ because $|\Delta| \rightarrow 0$ near the ends of the homogenous bridge where the cooling effect is weaker due to the diffusion of nonequilibrium quasiparticles to the leads. 


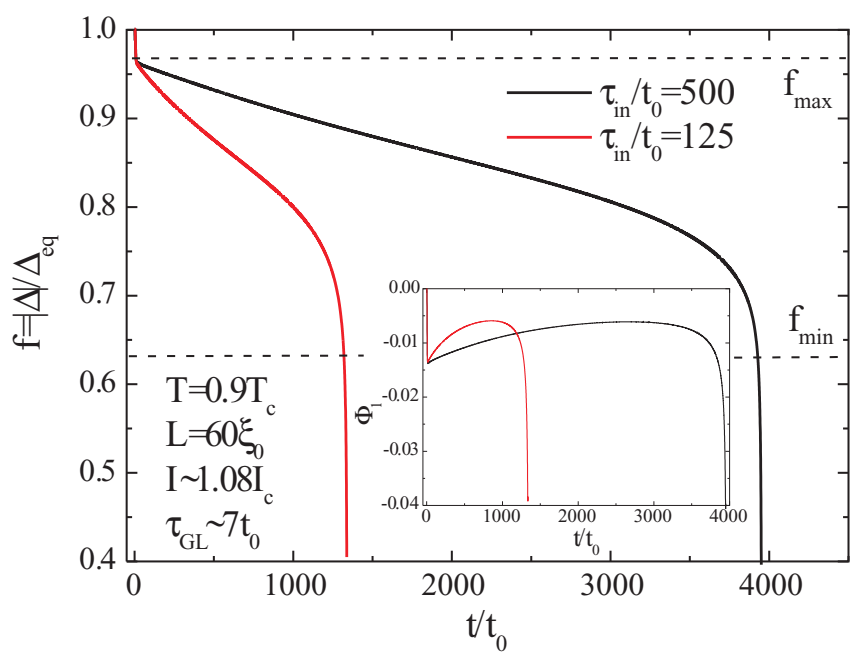

FIG. 2. (Color online) Time evolution of normalized $|\Delta|$ in the center of the bridge with a weak defect after the application of the supercritical current at $t=0$. Dashed lines show values of $f_{\max }$ and $f_{\text {min }}$ from Eqs. (11) and (12). In the inset we show the time dependence of $\Phi_{1}$ in the center of the bridge. Dynamics of both $|\Delta|$ and $\Phi_{1}$ does not depend on $\tau_{\text {in }}$ in the beginning and in the end of the transition process.

on the position, where $|\Delta|$ locally decays, with respect to the ends of the bridge (compare $t_{d}$ for an uniform bridge and for the bridge with a weak defect in Fig. 1) and on the length of the bridge when $L / 2 \lesssim L_{\text {in }}=\left(D \tau_{\text {in }}\right)^{1 / 2}$ (see Fig. 4).

In the model described by Eq. (13) $t_{d} \rightarrow 0$ when $f_{\text {min }}=$ $f_{\text {max }}$ which occurs for some current $I_{c 1}$ as given in Ref. [2]. Spatially nonuniform decay of $|\Delta|$ does not change this result qualitatively - the time delay drops fast when $I \rightarrow I_{c 1}$ (but its value is larger in the uniform model; see the inset in Fig. 3) while at larger currents $t_{d}$ is still finite and it does not depend on

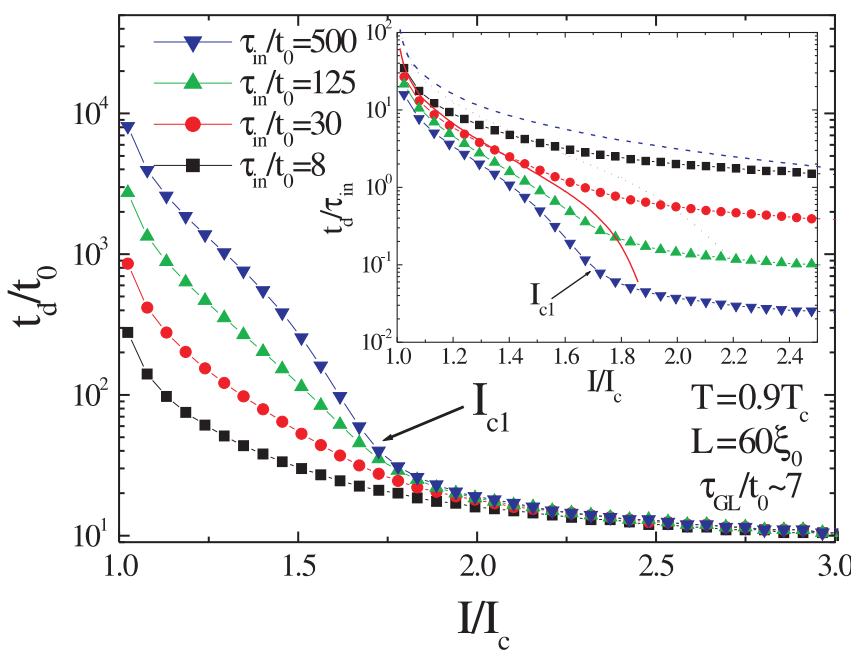

FIG. 3. (Color online) Dependence of the time delay on the normalized current for different $\tau_{\text {in }}$ (both comparable and much larger than $\tau_{G L}$ ). In the inset we show the same dependencies but with $t_{d}$ normalized in units of $\tau_{\text {in }}$. The solid curve corresponds to Eq. (13), the dotted curve to Eq. (13) with $Y(f)=1$ [Eq. (64) in Ref. [2]], and the dashed curve from Eq. (60) of Ref. [2] (local equilibrium limit).

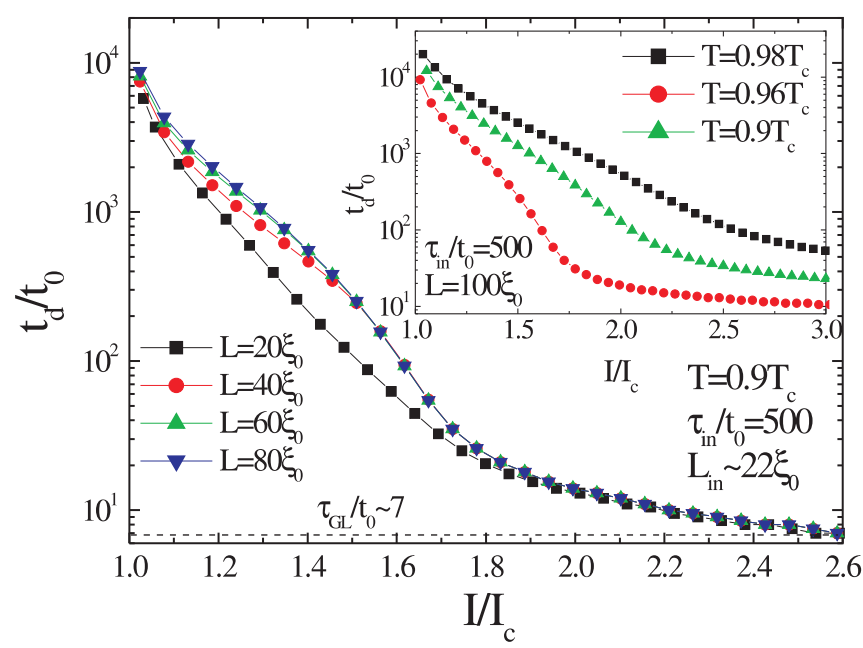

FIG. 4. (Color online) Dependence of the time delay on the normalized current for bridges with different lengths. For chosen $\tau_{\text {in }} / t_{0}=500$ and $L_{\text {in }} / \xi_{0} \simeq 22 \xi_{0}$ there is a strong decrease of $t_{d}$ when $L \lesssim 2 L_{\text {in }} \sim 40 \xi_{0}$ which we explain by increased diffusion of nonequilibrium quasiparticles to the leads. In the inset we show the dependence of $t_{d}$ on temperature for a long bridge $L \simeq 4.5 L_{\text {in }}$.

$\tau_{\text {in }}$ anymore. From a physical point of view for currents $I>I_{c 1}$ "cooling" of quasiparticles (which is limited by a decrease of $|\Delta|$ from $\Delta_{e q}$ to zero) cannot compensate the depairing effect of the current and the time delay does not depend on $\tau_{\text {in }}$. Finite delay time at $I>I_{c 1}$ is explained by the finite time $\tau_{J}=$ $\tau_{G L} / u\left[u \sim \pi^{4} / 14 \zeta(3)\right][25]$ needed for the transformation of the normal current to the superconducting one (assuming that the normal current appears in the bridge on a time scale much smaller than $\tau_{J}$ ) and finite relaxation time of $|\Delta|$, which imposes a relatively weak dependence on the current $t_{d}(I) \sim$ $\tau_{G L} I_{c} / I$.

Notice that not only diffusion of nonequilibrium quasiparticles is responsible for the reduction of $t_{d}$. It turns out that if we remove the term proportional to $q_{s}^{2}$ in Eq. (4) then the time delay and the current $I_{c 1}$ increases (but $t_{d}$ will still be smaller than in the uniform case). Finite $q_{s}$ smears out the peak in the density of states $N_{1}$ and in the spectral function $R_{2}$ at $\epsilon=|\Delta|[26]$ which results in a somewhat smaller value of $\Phi_{1}$, in comparison with the case when $q_{s}=0$, and thus smaller cooling effect.

Let us now discuss how the nonequilibrium contribution to the supercurrent $\delta j_{s}$ influences the time delay. From Fig. 5 one can see that finite $\delta j_{s} \neq 0$ leads to larger $t_{d}$ and $I_{c 1}$. In some respects finite $\delta j_{s}$ "compensates" the reduction of $t_{d}$ due to diffusion of quasiparticles and smearing of the spectral functions near $\epsilon=|\Delta|$ and shifts $t_{d}$ closer to the result expected from the uniform model. Originally the increase of $t_{d}$ due to finite $\delta j_{s}$ was predicted in Ref. [3] but the authors used a spatially uniform model and their values of $t_{d}$ were larger than Tinkham's result with $\delta j_{s}=0$ (see Fig. 5 in Ref. [2]) which is shown in Fig. 5 by the dashed curve.

\section{B. Quasiequilibrium model}

In the uniform case, from Eqs. (6) and (7) follows the equation for the time dependence of $f=|\Delta| / \Delta_{e q}$ which is 


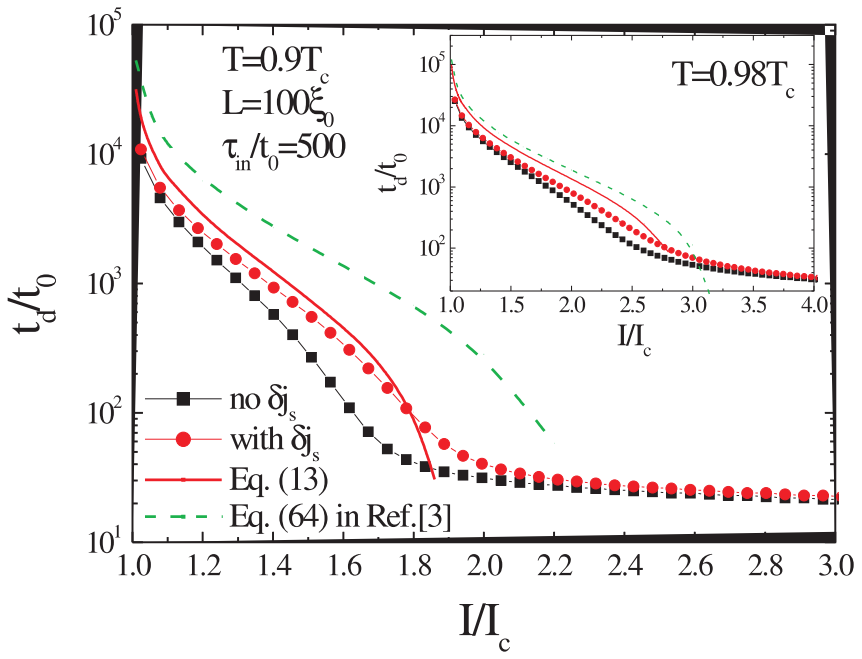

FIG. 5. (Color online) Change in the time delay when one takes into account the nonequilibrium contribution to the superconducting current. From the inset one can see that at higher temperature the effect of $\delta j_{s} \neq 0$ becomes smaller.

similar to Eq. (8),

$\tau_{G L} \frac{\partial f}{\partial t}+a_{T} f \int_{0}^{t} \frac{\partial f^{2}}{\partial t^{\prime}} e^{-\left(t-t^{\prime}\right) / \tau_{\mathrm{in}}} d t^{\prime}=f\left(1-f^{2}-\frac{\tilde{I}_{s}^{2}}{f^{4}}\right)$,

where $a_{T}=3 \Delta_{G L}^{2} / 2 \pi^{2} k_{B}^{2} T_{c}^{2} \simeq 1.42$. Again, when $\tau_{G L} \ll \tau_{\text {in }}$ we neglect the first term on the LHS of Eq. (14) and we find the differential equation

$$
\tau_{\text {in }} \frac{\partial f}{\partial t}=\frac{1-f^{2}-\tilde{I}_{s}^{2} / f^{4}}{2 f\left(1+a_{T}\right)-4 \tilde{I}_{s}^{2} / f^{5}} .
$$

Similar to the case which is considered in Sec. III A, Eq. (15) is not valid at the very beginning and at the end of the transition period when the denominator of Eq. (15) goes to zero. From Eq. (15) one may find the time delay

$$
\frac{t_{d}}{\tau_{\text {in }}}=\int_{f_{\max }}^{f_{\min }} \frac{2 f\left(1+a_{T}\right)-4 \tilde{I}^{2} / f^{5}}{1-f^{2}-\tilde{I}^{2} / f^{4}} d f,
$$

where $f_{\min }=\left[2 \tilde{I}^{2} /\left(1+a_{T}\right)\right]^{1 / 6}$ and $f_{\max }$ should be found from the equation

$$
\tilde{I}^{2}=f_{\max }^{4}\left(1+a_{T}\right)\left(1-f_{\max }^{2}\right) .
$$

From Eq. (16) it follows that $t_{d}$ depends only on temperature via $\tau_{\text {in }}(T)$. This is the main qualitative difference with Eq. (13), where the strong temperature dependence of $t_{d}$ comes from $a(T)$ and $Y(T)$. As a consequence, in the quasiequilibrium model the current $I_{c 1} \simeq 1.55 I_{c}$ (when $f_{\text {max }}=f_{\text {min }}$ and $t_{d} \rightarrow 0$ ).

In Fig. 6 we present the dependence $t_{d}\left(I / I_{c}\right)$ for a bridge with length $L=100 \xi_{0}$ found from a numerical solution of Eqs. (6) and (7) and $\operatorname{div} j=0$. As in the nonthermal model the effect of quasiparticle diffusion is large and in the homogenous bridge the order parameter decays faster near the leads. Therefore we suppress $T_{c}$ in the center of the bridge by $4 \%$ in the region with size $\xi_{0}$ and in Fig. 6 we show the time decay

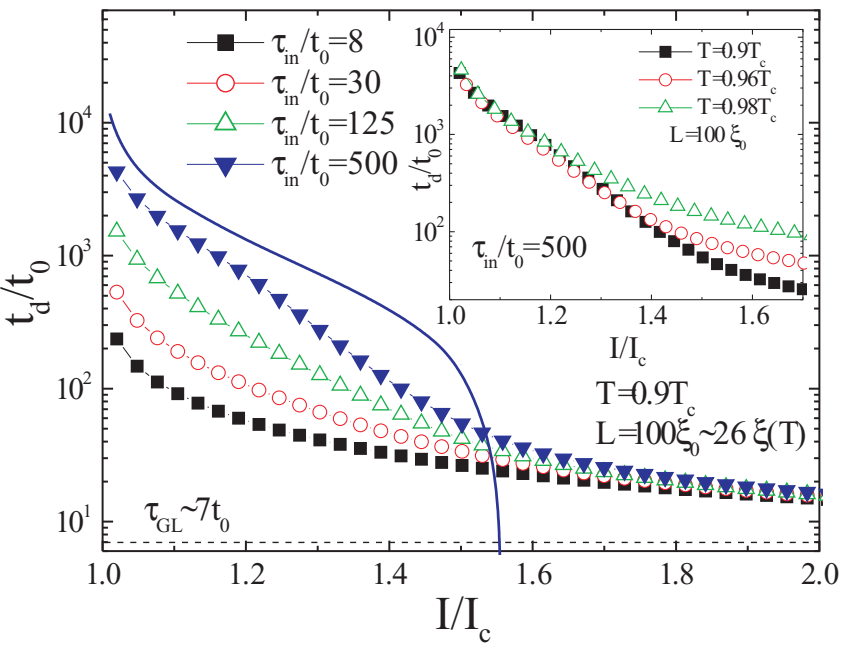

FIG. 6. (Color online) Dependence of the time delay on the normalized current for a superconducting bridge (quasiequilibrium model). The solid curve corresponds to Eq. (16) with $\tau_{\text {in }} / t_{0}=500$. In the inset we show the dependence $t_{d}\left(I / I_{c}\right)$ for different temperatures.

of the order parameter in this place. In the inset one can see rather weak temperature dependence of $t_{d}$ at currents near $I_{c}$ and strong temperature dependence at $I \gtrsim I_{c 1} \sim 1.55 I_{c}$. The latter occurs due to the strong temperature dependence of $\tau_{G L}$ which mainly determines the time delay at $I \gtrsim I_{c 1}$.

From a comparison of Figs. 3 and 6 one can see that the dependence of the time delay on current and $\tau_{\text {in }}$ in the quasiequilibrium limit resembles the one in the nonthermal limit but it is shorter for fixed $I / I_{c}$ and $\tau_{\text {in }}$. We can explain this as follows. In the nonthermal model the cooling effect comes from the term $R_{2} \partial|\Delta| / \partial t$ in the kinetic equation which is peaked at energies $\epsilon \simeq|\Delta|$ and it has a long tail $\sim|\Delta| / \epsilon$ at larger energies. It results in the largest deviation from equilibrium at $\epsilon \simeq|\Delta|$ and this interval of energies gives a relatively large contribution to $\Phi_{1}$. In the quasiequilibrium model, due to the thermalization process this peak is smeared out and it leads to a smaller value of $\Phi_{1}$ and a weaker cooling effect.

\section{DYNAMIC RESPONSE IN A 2D BRIDGE}

Now we study the dynamic response in a two-dimensional bridge with nonuniform current distribution across the superconductor. In our case the nonuniformity arises due to the application of a perpendicular magnetic field (see Fig. 7). We consider only weak fields when there are no vortices in the bridge at currents below $I_{c}$.

Due to the nonuniform current distribution the order parameter decays first near the edge where the current density and supervelocity are maximal. When near the edge $|\Delta| \rightarrow 0$ the vortex enters the film and passes through it (for our length of the bridge only one vortex enters simultaneously). Behind the moving vortex there is a wake-a region with suppressed order parameter [see inset (b) in Fig. 8] which appears due to the large recovery time of $|\Delta|$ (in the local equilibrium approximation, when $\tau_{\text {in }}<\tau_{G L}$, this effect was studied in Refs. [27,28]). This wake favors faster nucleation of the second and subsequent 


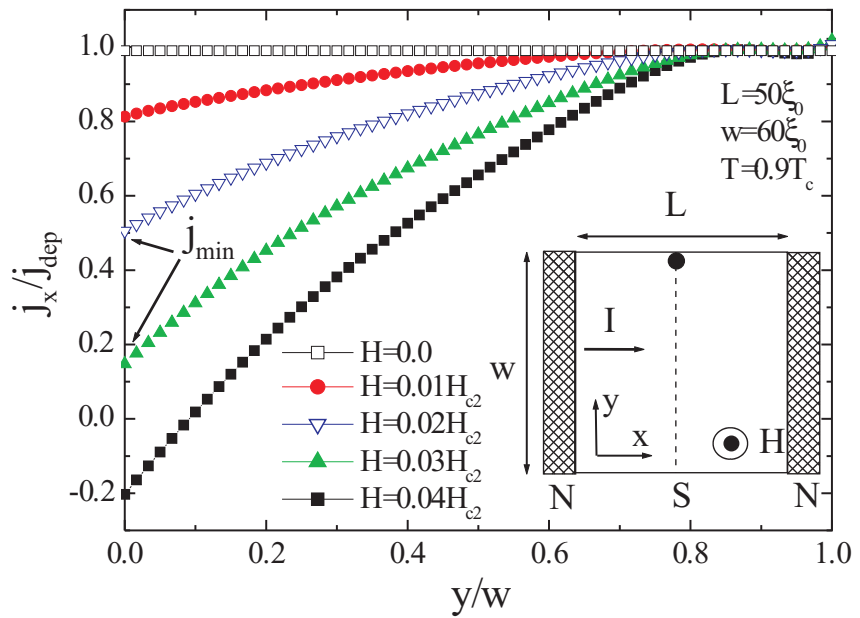

FIG. 7. (Color online) Distribution of the current density across the superconducting bridge (along the dashed line in the sketch of the bridge shown in the inset) at different magnetic fields and currents just below $I_{c}(H)$. In the inset we show a sketch of the bridge contacted to normal leads. To prevent the influence of nonequilibrium effects from the NS boundaries we locally (on the distance $5 \xi_{0}$ from each end) increased the local $T_{c}$ by $20 \%$.

vortices [in Fig. 8 every minimum in $|\Delta|(t)$ corresponds to the entrance of a new vortex] because of subsequent gradual suppression of $|\Delta|$ (see Fig. 8). Passage of several vortices nucleates a quasiphase slip line [28] - the region with width $\sim 2 \xi$ where $|\Delta|$ is strongly suppressed but is still different from zero [see insets (c) and (d) in Fig. 8]. This quasiphase slip line (PSL) may convert or not to a normal domain which then spreads over the superconductor if the current or $\tau_{\text {in }}$ is large enough. Note that in the present model only partial Joule dissipation is considered (via the dependence of the Green

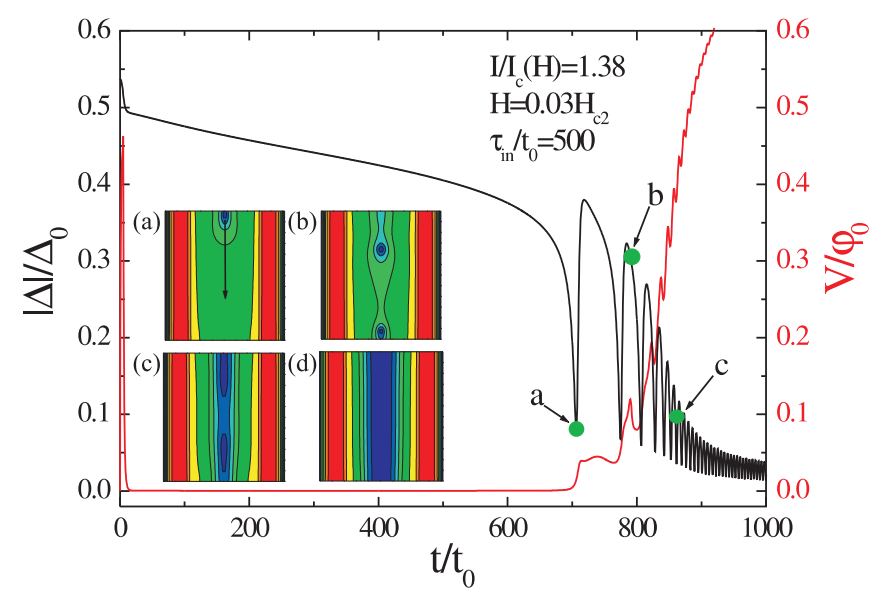

FIG. 8. (Color online) Time dependence of $|\Delta|$ at the edge of the bridge (marked as black spot in the inset in Fig. 7) and voltage drop across the bridge at $I / I_{c}(H) \simeq 1.38$ and $H=0.03 H_{c 2}$ (nonthermal model). In the inset we present snapshots of $|\Delta|$ in the bridge at different moments in time: (a) $t / t_{0}=706$, (b) $t / t_{0}=789$, (c) $t / t_{0}=863$, and (d) $t / t_{0}=1500$. Arrow in inset (a) shows the direction of vortex motion. The narrow peak in the voltage at $t \simeq 0$ is connected with initially normal current $I_{n}=I$ which transforms to the superconducting one on the time scale $\tau_{J}$.

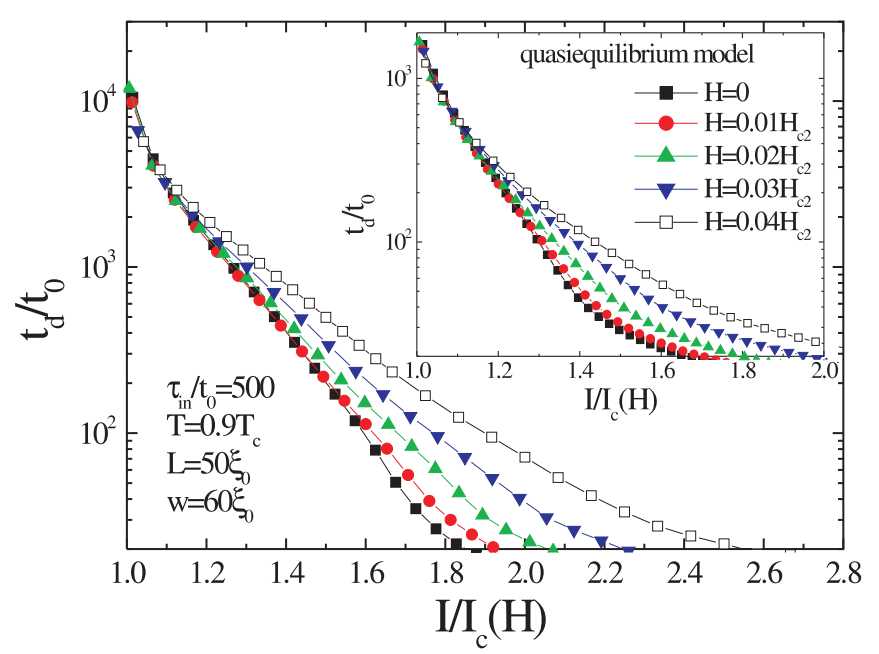

FIG. 9. (Color online) Dependence of the time delay (in the appearance of the first vortex) on the applied current at different magnetic fields (nonthermal model). In the inset we show the same dependencies calculated in the quasiequilibrium model.

functions on the supervelocity; see Ref. [29]) and hence the time for nucleation of the quasi-PSL is underestimated.

When the ratio $j_{\min } / j_{\text {dep }}$ is small (for definition of $j_{\min }$ see Fig. 7) the quasi-PSL may not appear at $I \sim I_{c}(H)$ because in the part of the film where $j$ is small the vortices move slowly and $|\Delta|$ has time to recover. Whether quasi-PSL appears or not at $I=I_{c}(H)$ is controlled by $\tau_{\text {in }}$ - the larger $\tau_{\text {in }}$ the smaller the current threshold value $I_{t h}\left(\tau_{\text {in }}\right)$ when quasi-PSL appears in the bridge [28]. If $I_{t h}>I_{c}(H)$, then in the current interval $I_{c}(H)<I<I_{t h}$ there is slow vortex motion and the voltage signal is small, while for $I>I_{t h}$ there will be a sudden jump in the voltage connected with the appearance of the quasi-PSL.

Below we consider the situation when $I_{c}(H)>I_{t h}$. Due to the nonuniform (over width of the bridge) decay of $|\Delta|$ there is an uncertainty in the definition of the time delay. One of the variants is to define it as the time needed for the nucleation of the first vortex after the application of the supercritical current (this time is shown in Fig. 9). From Fig. 8 it is clear that this definition underestimates $t_{d}$ because a large voltage response appears only after the nucleation of the quasi-PSL. But a noticeable difference arises only at relatively large currents when these two times become comparable with each other (for example, for the parameters of Fig. 8 the first vortex nucleation time is $\sim 700 t_{0}$, while the PSL nucleation time is $\sim 900 t_{0}$ ).

Our simulations show that the delay in the appearance of the quasiphase slip line after the nucleation of the first vortex is mainly determined by the "flight" time of the first vortex across the bridge. We find that the second, third, and so on vortices enter the bridge before the first vortex exits. The number of vortices simultaneously present in the bridge depends on the width of the bridge, $\tau_{\text {in }}$, and on the ratio $j_{\min } / j_{\text {dep }}$. For example, in the bridge with $w=50 \xi_{0}$ there are two vortices in the quasiphase slip line [see insets (b) and (c) in Fig. 8], while in the bridge with $w=120 \xi_{0}$ there are already four vortices (for the same $\tau_{\text {in }}$ and ratio $j_{\min } / j_{\text {dep }}$ ).

The flight time of the first vortex $t_{f}$ could be roughly estimated using results for the time delay in quasi-1D bridges 
and the knowledge about the basic properties of the vortex. First of all one should be reminded that next to the vortex core the superconducting current density $\sim j_{\text {dep }}$. From one side of the vortex this current density and the transport current density are summed up and it leads to a local destruction of superconductivity and vortex motion in that direction. If the sum of these current densities exceeds $j_{c 1}=I_{c 1} / w d$, then the time decay of $|\Delta|$ is rather short $\left(t_{d} \sim \tau_{G L} I_{\mathrm{dep}} / I\right.$; see Sec. III) and the first vortex moves fast. One can estimate its average velocity as $v_{\text {aver }} \sim \xi(T) / t_{d}$. For our bridge with $w=60 \xi_{0} \simeq 16 \xi\left(T=0.9 T_{c}\right)$ the flight time of the first vortex is about $t_{f} \simeq w / v_{\text {aver }} \sim 16 \tau_{G L} \sim 112 t_{0}$ (for parameters of Fig. 8 where $\left.I_{c}\left(H=0.03 H_{c 2}\right)=0.75 I_{\text {dep }}\right)$ which is not far from the numerical value $\sim 83 t_{0}$.

As the current approaches $I_{c}$ the nucleation time of the first vortex increases much faster than the first vortex flight time and the time delay is mainly determined by the former time (except for very wide films with $w \gg \xi t_{d} / \tau_{G L} \sim \xi \tau_{\text {in }} / \tau_{G L}$ ).

The above rough estimations are valid if the sum of the current densities from the vortex and the transport current exceeds $j_{c 1}$. In the case of a small ratio $j_{\text {min }} / j_{\text {dep }}$ this condition is not fulfilled and the first vortex moves with a much lower velocity. As a limiting case the quasi-PSL is not nucleated and at $I \sim I_{c}$ there is only slow vortex motion.

In the quasiequilibrium limit we have qualitatively the same results for the dependence of $t_{d}$ (time nucleation of the first vortex) on the applied magnetic field (see inset in Fig. 9). Note that in both models at $I \simeq I_{c}(H)$ this time delay slightly decreases with an increase of $H$ (probably it is connected with a stronger diffusion of the nonequilibrium quasiparticles in the $2 \mathrm{D}$ case in comparison with 1D) while at larger currents $t_{d}$ increases [for the same ratio $I / I_{c}(H)$ ]. The last effect can be connected with the current redistribution when $|\Delta|$ becomes locally suppressed near one edge during the transition and the superconducting current escapes that region which leads to a locally smaller value of the current density.

\section{DYNAMIC RESPONSE ON AN ALTERNATING CURRENT PULSE}

In this section we study the dynamic response of the superconducting bridge on an alternating current pulse with amplitude $I_{\mathrm{ac}}$ larger than $I_{c}$ and with a zero time average [the ac current pulse is modeled as one period of the sinusoid $\left.I=I_{\mathrm{ac}} \sin \left(2 \pi t / T_{\mathrm{ac}}\right)\right]$. Our interest in this problem arises from recent work [16] where the voltage response of the superconducting YBCO bridge on a short pulse of synchrotron radiation (with a duration of several picoseconds) was experimentally observed even in the absence of dc current. Due to the absence of a bolometric origin of the resistive response authors supposed that the electric field of the electromagnetic radiation accelerates the superconducting electrons and when the radiation induced current exceeds the critical current a finite voltage appears in the bridge.

Based on the results of Secs. III-IV we may give the following rough criterion: At given amplitude $I_{\mathrm{ac}}$ a resistive response does exist when during the ac current pulse the time interval when $|I|>I_{c}$ exceeds $t_{d}\left(I_{\mathrm{ac}}\right)$, where $t_{d}\left(I_{\mathrm{ac}}\right)$ corresponds to the time delay on the abrupt switching on of the dc current with amplitude $I_{\mathrm{ac}}$. We numerically checked

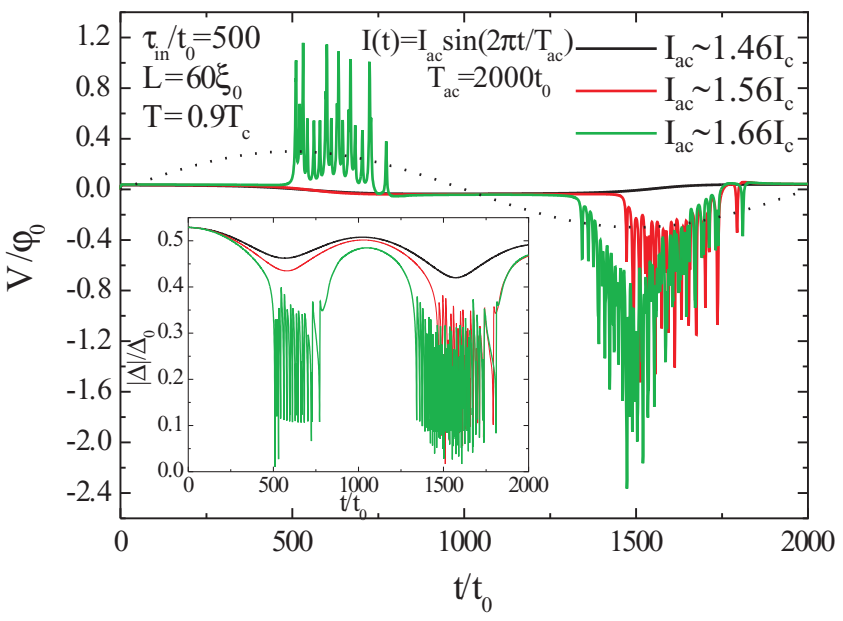

FIG. 10. (Color online) Time dependence of the voltage in the 2D superconducting bridge during an ac current pulse (marked as a dotted line). In a relatively narrow range of amplitudes of the current pulse the larger voltage appears only for one direction of the current. In the inset we show the time dependence of the order parameter in the center of the bridge. Calculations are made within the nonthermal model.

and confirmed this idea on the example of a quasi-1D superconducting bridge. We also find one interesting effect which arises when the amplitude of the ac pulse approaches some critical value. From Fig. 10 one can see that with increasing $I_{\mathrm{ac}}$ a large voltage appears first in the second half of the ac current pulse and one needs to increase $I_{\mathrm{ac}}$ to observe it in the first half too. We explain this effect as follows. During the first half of the pulse the order parameter is getting suppressed and it does not recover its equilibrium value at $t=T_{\mathrm{ac}} / 2$ [when

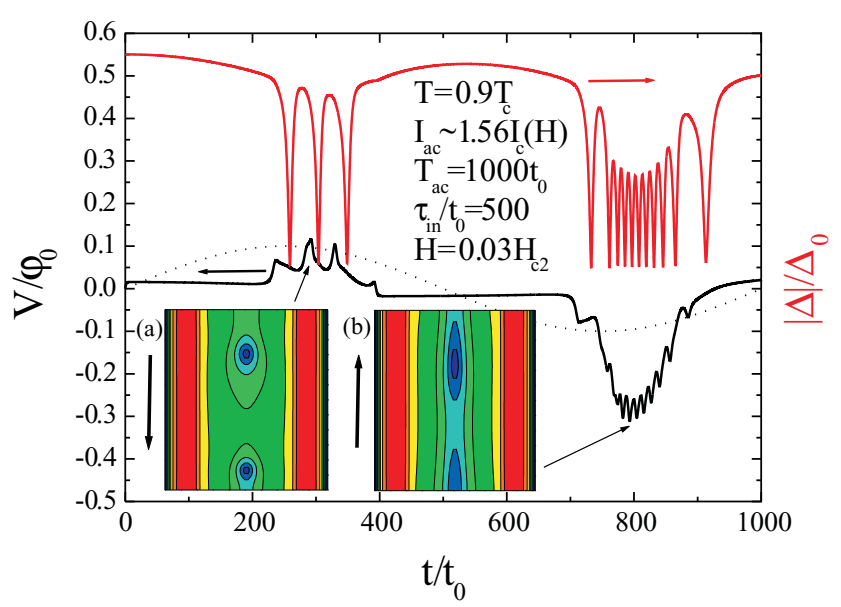

FIG. 11. (Color online) Time dependence of the voltage across the bridge and magnitude of order parameter in the center of superconductor when ac current pulse (marked as a dotted line) is applied. In the inset we show spatial distribution of $|\Delta|$ at $t=290 t_{0}$ (a) and at $t=800 t_{0}$. In the second half of the pulse the order parameter is suppressed strongly along the path of vortex motion which ensures larger vortex velocity and higher value of the voltage than in the first half of the current pulse. Calculations are made in quasiequilibrium model. 
$I(t)=0]$ due to the finite relaxation time of $|\Delta|$ (see the inset in Fig. 10 for $I=1.46 I_{c}$ and $\left.I=1.56 I_{c}\right)$. At $T_{\mathrm{ac}} / 2<t<T_{\mathrm{ac}}$ there is further suppression of $|\Delta|$ and it goes to zero (when $I_{\mathrm{ac}}$ is sufficiently strong) and a highly resistive state appears in the bridge (in the used 1D bridge it is realized as a phase slip process). As a result the time-averaged voltage is not equal to zero and its sign depends on the phase of the ac current pulse-with change of the phase by $\pi$ the sign of the time-averaged voltage changes.

Dynamic response of a 2D bridge with nonuniform current distribution is qualitatively similar to the case of a 1D bridge. When the amplitude of the ac pulse exceeds some critical value (at fixed period $T_{\mathrm{ac}}$ ) the vortices enter the bridge one by one and they suppress $|\Delta|$ along their trajectory of motion (see Fig. 11). At $t=T_{\mathrm{ac}} / 2$ there are no vortices in the bridge (for the chosen parameters in Fig. 11) but $|\Delta|$ is suppressed below its equilibrium value and it facilitates the faster vortex motion in the second half of the pulse. As a result the voltage is larger in the second half of the pulse (see Fig. 11).

\section{DISCUSSION}

In the first experiment on transient response it was found that the time delay in Al films does not depend on temperature when $0.76<T / T_{c}<0.92$ [1]. Subsequent experiments on $\mathrm{Al}$ [5-7] and In [4] films found a strong temperature dependence of $t_{d}$ near $T_{c}$. A later experiment on a YBCO bridge again did not reveal any temperature dependence of $t_{d}$ in a wide temperature interval 4.2-68 K [8]. Note that all previous theories predicted a strong temperature dependence of $t_{d}$ near $T_{c}$ [2,3] due to the coefficient $a(T)$ in Eq. (13). In Ref. [8] authors tried to resolve this problem by the replacement of the product $a(T) \tau_{\text {in }} \sim \tau_{|\Delta|}$ by a temperature-independent escape time of nonequilibrium phonons $\tau_{e s c}$ to the substrate. Our results give physical and mathematical reasons for such a replacement. In a case in which the escape time of phonons to the substrate is the longest relaxation time the electrons and phonons have one temperature which is different from the bath temperature. In this case one should use the quasiequilibrium model with $\tau_{\text {in }}=\tau_{\text {esc }}$ and this model predicts a temperatureindependent $t_{d}\left[I / I_{c}(T)\right]$ at currents relatively close to $I_{c}$ [see the inset in Fig. 6 and Eq. (16)].

In the case when there is good thermal connection between the superconductor and the substrate and $\tau_{e s c} \ll \tau_{e-p h} \ll \tau_{e-e}$ the nonthermal model is more relevant. This model predicts strong temperature dependence of $t_{d}$ near $T_{c}$ (see inset in Fig. 4) which is even stronger than that following from Refs. [2,3] because of the temperature-dependent $Y(T)$. In a more complicated situation the times $\tau_{e-p h}, \tau_{e-e}$, and $\tau_{e s c}$ could be comparable with each other which brings a more complex temperature dependence of $t_{d}(T)$ (it should be something in between the two limiting cases considered here).

In our calculations we neglect the temperature dependence of $\tau_{\text {in }}$. It is approximately valid when $\tau_{\text {in }}=\tau_{\text {esc }}$ and not in the case when $\tau_{\text {in }}=\tau_{e-p h} \sim 1 / T^{3}$ (this temperature dependence comes from the Debye model of phonons). However, if one does not go far from $T_{c}$ it gives only small corrections in $\tau_{e-p h}\left[\right.$ for example, $\left.\tau_{e-p h}\left(0.9 T_{c}\right) \simeq 1.4 \tau_{e-p h}\left(T_{c}\right)\right]$.

How is the time delay modified when we consider low temperatures? At low temperatures there is an exponential decay of $Y(T)$ when $k_{B} T \ll \Delta_{e q}$ [which follows from the general expression for $Y(T)$; see Eq. (9)] and it formally leads to a fast decrease of $t_{d}$ when $T \rightarrow 0$ despite the increase of $\tau_{\text {in }}=\tau_{e-p h}$ (in the nonthermal model where the phonons are in equilibrium). But one should remember that Eqs. (2)-(5) were derived at $T \sim T_{c}$ (when $\Delta_{e q} \ll k_{B} T$ ) and many terms were omitted, which can become important at low temperatures. Therefore this question needs additional investigation both in nonthermal and quasiequilibrium limits.

Our predictions for the dependence of the time delay of narrow superconducitng bridges/films (with width $\xi \ll w \ll$ $\lambda^{2} / d$ ) on the applied magnetic field could be checked by an experiment. We predict that in relatively weak magnetic fields $0<H \lesssim \Phi_{0} / 4 \pi \xi w$ (when the superconductor is in the Meissner state) the time delay depends weakly on $H$ for $I \gtrsim$ $I_{c}(H)$ and for larger currents $I \gg I_{c}(H), t_{d}$ should increase with increasing $H$. The crucial effect for the observation of long delay times at $I \sim I_{c}(H)$ is the absence of trapped vortices in the superconductor. Indeed, our calculations show that for not very wide films $w \ll \xi t_{d} / \tau_{G L} \sim \xi \tau_{\text {in }} / \tau_{G L}$ the time delay is determined mainly by the nucleation time of the first vortex and the presence of trapped vortices should decrease $t_{d}$.

Our theoretical results for the dynamic response of a superconducting bridge on the short ac current pulse qualitatively resembles some of the experimental results of Ref. [16]. Namely, we also find nonzero voltage response in the absence of a dc current and a change of its sign when the phase of the ac current changes by $\pi$. However, we use a very simple shape of the ac current pulse (sinusoid) which is drastically different from the asymmetric pulse in the experiment (see Fig. 9 in Ref. [16]). If the pulse is asymmetric (the amplitude of the current of one sign is much larger than the amplitude of the current of the opposite sign), then the sign of the voltage response is determined by the largest current in the pulse. This makes it difficult to directly compare our results with those of Ref. [16] but in any case we predict that the response may appear only if the duration of the pulse is larger than the time delay at the largest amplitude of the current pulse.

In Ref. [16] no external magnetic field was present but the current distribution probably was nonuniform across the bridge both due to current-crowding effect at the ends of the bridge [15] and the small aspect ratio $L / w \sim 0.44$ [30]. Indirectly it could be seen from the measured current-voltage $(I V)$ characteristic which has a low voltage tail at currents close to $I_{c}$ and a sharp voltage jump at large currents (see Fig. 4 in Ref. [16]) which resembles the $I V$ curves of wide superconducting bridges with $w \gg \lambda^{2} / d$ (see Fig. 2 in [31]). The width of the used bridge $(w=4.5 \mu \mathrm{m})$ satisfies the condition $w \gg \xi \tau_{\text {in }} / \tau_{G L}$ [where we use $\tau_{\text {in }} \sim 1.7 \times 10^{-11} \mathrm{~s}$ at $T=0.9 T_{c}$ [32] and $\xi\left(T=0.9 T_{c}\right) \sim 5 \mathrm{~nm}$ ] which means that the main contribution to the time delay comes from the forming of a quasiphase slip line. It is clear that the last time depends not only on the intrinsic parameters of the superconductor $\left(\tau_{\text {in }}\right)$ but also on the width of the bridge.

\section{CONCLUSION}

In our work we study the dynamic response of 1D and 2D superconducting bridges after the abrupt switching on of the supercritical current. We present calculations near 
the critical temperature of the superconductor in two limits: (i) nonthermal limit, when the energy relaxation time of electrons due to electron-phonon interaction is the shortest one and the phonons are assumed to be in equilibrium, and (ii) quasiequilibrium limit when the energy relaxation time of electrons is determined by the escape time of the nonequilibrium phonons to the substrate and one can use the local temperature approach. We find that in both limits the fastest decay of the superconducitng order parameter occurs near the ends of the homogenous bridge or, for a weakly inhomogenous bridge, in defect places where the local critical current is smallest. We find that the time decay of $|\Delta|$ is smaller than in the model with spatially uniform suppression of $|\Delta|$ due to the diffusion of nonequilibrium ("cooled") quasiparticles from the region where $|\Delta|$ decays faster. Smearing of the density of states and the spectral functions at energies close to $|\Delta|$ (arising from finite supervelocity) is another factor which leads to a decrease of $t_{d}$. Time delay does not depend on the temperature in the quasiequilibrium limit (at currents slightly exceeding $I_{c}$ ) which is in strict contrast with the nonthermal model which predicts a strong temperature dependence of $t_{d}$ taken at the same values $I / I_{c}(T)$.

Dynamic response of the 2D bridge with nonuniform current distribution resembles the response of the bridge with uniformly distributed current but it has also some qualitative differences. The superconducting state in a $2 \mathrm{D}$ bridge is destroyed by the appearance of vortices and the subsequent nucleation of the quasiphase slip line across the bridge. For not very wide films $w / \xi \lesssim \tau_{\text {in }} / \tau_{G L}$ the nucleation time of the first vortex and nucleation time of the quasiphase slip line are close to each other at currents not far from $I_{c}$. By varying the weak applied magnetic field one may change the level of nonuniformity in the current distribution and tune the time delay.

A voltage response of the superconducting bridge on an alternating current pulse (sinusoidlike) is predicted if the duration of the pulse is larger than the time delay after the abrupt switching of the current with magnitude equal to the amplitude of the ac current pulse. We also find that the timeaveraged voltage is not zero despite the zero time-averaged current and its sign changes when the phase of the ac current is changed by $\pi$.

\section{ACKNOWLEDGMENTS}

This work was partially supported by the Russian Foundation for Basic Research (Project No. 12-02-00509), by the Ministry of Education and Science of the Russian Federation (the agreement of August 27, 2013, No. 02.B.49.21.0003, between The Ministry of Education and Science of the Russian Federation and Lobachevsky State University of Nizhni Novgorod) and by the European Science Foundation (ESF) within the framework of the activity entitled "Exploring the Physics of Small Devices (EPSD)" (Project No. 4327).

\section{APPENDIX: DERIVATION OF THE HEAT CONDUCTANCE EQUATION}

Here we derive the heat conductance equation which governs the dynamics of the local temperature of the quasiparticles in the quasiequilibrium limit when the electric field $E$ is small (or $E$ is finite only during a short time interval $\lesssim \tau_{G L}$ ) and one may neglect the Joule heating. First of all from Eq. (4) it follows that

$$
\frac{\partial N_{1}}{\partial t}+\frac{\partial|\Delta|}{\partial t} \frac{\partial R_{2}}{\partial \epsilon}-\frac{4 D q_{s}}{\hbar} \frac{\partial q_{s}}{\partial t} \frac{\partial R_{2} N_{2}}{\partial \epsilon}=0 .
$$

In the next step we multiply Eq. (A1) by $f_{L}$, add it to the equation for $f_{L}$ [Eq. (2)], and then multiply the final equation by $N_{0} \epsilon$ and integrate over the energy. As a result we obtain (with the help of the self-consistency equation $\Delta / v=1 / 2 \int R_{2} f_{L} d \epsilon$, where $v$ is a coupling constant) the equation for the energy balance (per unit volume)

$$
\begin{aligned}
\frac{\partial}{\partial t}[ & {\left[2 N_{0} \int_{-\infty}^{\infty} \epsilon N_{1} f_{L} d \epsilon-|\Delta|^{2} / \nu\right] } \\
= & 2 N_{0} D \nabla\left(\int_{-\infty}^{\infty} \epsilon\left(N_{1}^{2}-R_{2}^{2}\right) \nabla f_{L} d \epsilon\right) \\
& -\frac{2 N_{0}}{\tau_{\text {in }}} \int_{-\infty}^{\infty} \epsilon N_{1}\left(f_{L}-f_{L}^{0}\right) d \epsilon .
\end{aligned}
$$

In Eq. (A2) the term in brackets on the LHS corresponds to the energy of the electrons per unit volume $\mathcal{E}=F+T S$ ( $S$ is the entropy and $F$ is the free energy per unit volume). This equation [here with omitted terms which incorporate effects of Joule dissipation and coupling between transverse $f_{T}$ and longitudinal $f_{L}$ parts of $f(\epsilon)$ ] was originally derived in [21] [see Eq. (C.4) there]. We seek for the solution of this equation in the form

$$
f_{L}(\epsilon)=\tanh \left(\epsilon / 2 k_{B} T_{\mathrm{loc}}\right)
$$

and insert it in Eq. (A2). After integration over energy we find the equation for the temperature of quasiparticles

$$
\begin{aligned}
& \frac{\partial}{\partial t}\left(\frac{N_{0} \pi^{2} k_{B}^{2} T_{\mathrm{loc}}^{2}}{3}-N_{0}|\Delta|^{2} \frac{T_{\mathrm{loc}}}{T_{c}}\right) \\
& \quad=\frac{2 N_{0} D \pi^{2} k_{B}^{2}}{3} \nabla\left(T_{\mathrm{loc}} \nabla T_{\mathrm{loc}}\right)-\frac{N_{0} \pi^{2} k_{B}^{2}}{3} \frac{T_{\mathrm{loc}}^{2}-T^{2}}{\tau_{\mathrm{in}}} .
\end{aligned}
$$

In the derivation of Eq. (A4) we used the Ginzburg-Landau expression for the free energy $F$, the expression for the entropy $S$ of the superconductor near $T_{c}\left[S=2 \pi^{2} N(0) k_{B}^{2} T / 2-\right.$ $\left.N(0)|\Delta|^{2} / T_{c}\right]$ and assume that $\Delta$ satisfies the time-dependent Ginzburg-Landau equation [Eq. (3)]. We also neglect terms $\sim|\Delta|^{4}$ in $\mathcal{E}$ which leads to $\partial|\Delta|^{4} / \partial t \sim \Delta_{e q}^{4} / \tau_{G L} \sim(1-$ $\left.T / T_{c}\right)^{3}$ in the RHS of Eq. (A4) and which is small near $T_{c}$ [note that it has the same smallness as the neglected Joule dissipation $\left.j E \sim \sigma_{n} j_{\text {dep }}^{2} \sim\left(1-T / T_{c}\right)^{3}\right]$. In the framework of Eq. (A4) the cooling of quasiparticles due to a decreasing $|\Delta|$ has a simple physical origin-because of energy conservation the temperature of quasiparticles should go down to compensate the energy increase due to the suppression of $|\Delta|$.

When the deviation from the equilibrium temperature is small, $\left|T_{\text {loc }}-T\right|=\left|\delta T_{\text {loc }}\right| \ll T$, one may linearize Eq. (A4) and we arrive at Eq. (6). 
[1] J. A. Pals and J. Wolter, Phys. Lett. A 70, 150 (1979).

[2] M. Tinkham, in Nonequilibrium, Superconductivity, Phonons, and Kapitza Boundaries, Proceedings of NATO Advanced Study Institutes, edited by K. E. Gray (Plenum, New York, 1981), p. 231.

[3] A. Geier and G. Schön, J. Low Temp. Phys. 46, 151 (1982).

[4] D. J. Frank, M. Tinkham, A. Davidson, and S. M. Faris, Phys. Rev. Lett. 50, 1611 (1983).

[5] J. Wolter, P. M. Th. M. van Attekum, R. E. Horstman, and M. C. H. M. Wouters, Physica B+C 108, 781 (1981).

[6] P. M. Th. M. van Attekum, M. C. H. M. Wouters, J. Wolter, and R. E. Horstman, Solid State Commun. 40, 1019 (1981).

[7] J. Wolter, P. M. Th. M. van Attekum, R. E. Horstman, and M. C. H. M. Wouters, Solid State Commun. 40, 433 (1981).

[8] F. S. Jelila, J. P. Maneval, F. R. Ladan, F. Chibane, A. Mariede-Ficquelmont, L. Mechin, J. C. Villegier, M. Aprili, and J. Lesueur, Phys. Rev. Lett. 81, 1933 (1998).

[9] M. Tinkham, Introduction to Superconductivity (McGraw-Hill, New York, 1996).

[10] K. E. Nagaev, Phys. Rev. B 52, 4740 (1995).

[11] F. Giazotto, T. T. Heikkilä, A. Luukanen, A. M. Savin, and J. P. Pekola, Rev. Mod. Phys. 78, 217 (2006).

[12] A. Buzdin and M. Daumens, Physica C 294, 257 (1998).

[13] A. Yu. Aladyshkin, A. S. Mel'nikov, I. A. Shereshevsky, and I. D. Tokman, Physica C 361, 67 (2001).

[14] D. Yu. Vodolazov, Phys. Rev. B 62, 8691 (2000).

[15] J. R. Clem and K. K. Berggren, Phys. Rev. B 84, 174510 (2011).

[16] P. Probst, A. Semenov, M. Ries, A. Hoehl, P. Rieger, A. Scheuring, V. Judin, S. Wünsch, K. Il'in, N. Smale, Y.-L. Mathis, R. Müller, G. Ulm, G. Wüstefeld, H.-W. Hübers, J. Hänisch, B. Holzapfel, M. Siegel, and A.-S. Müller, Phys. Rev. B 85, 174511 (2012).
[17] A. Schmid and G. Schön, J. Low Temp. Phys. 20, 207 (1975).

[18] A. I. Larkin and Yu. N. Ovchinnikov, Zh. Eksp. Teor. Fiz. 73, 299 (1977) [Sov. Phys. JETP 73, 155 (1977)].

[19] L. Kramer and R. J. Watts-Tobin, Phys. Rev. Lett. 40, 1041 (1978).

[20] R. J. Watts-Tobin, Y. Krähenbühl, and L. Kramer, J. Low Temp. Phys. 42, 459 (1981).

[21] A. Schmid, in Nonequilibrium Superconductivity, Phonons, and Kapitza Boundaries, edited by K. E. Gray (Plenum, New York, 1981), p. 423.

[22] Th. Y. Hsiang and J. Clarke, Phys. Rev. B 21, 945 (1980).

[23] V. P. Galaiko, Zh. Eksp. Teor. Fiz. 68, 223 (1975) [Sov. Phys. JETP 41, 108 (1975)].

[24] I. F. Oppenheim, S. Frota-Pessôa, and M. Octavio, Phys. Rev. B 25, 4495 (1982).

[25] B. I. Ivlev and N. B. Kopnin, Adv. Phys. 33, 47 (1984).

[26] A. Anthore, H. Pothier, and D. Esteve, Phys. Rev. Lett. 90, 127001 (2003).

[27] L. I. Glazman, Fiz. Nizk. Temp. 12, 688 (1986) [Sov. J. Low Temp. Phys. 12, 389 (1986)].

[28] D. Yu. Vodolazov and F. M. Peeters, Phys. Rev. B 76, 014521 (2007).

[29] D. Y. Vodolazov and F. M. Peeters, Phys. Rev. B 83, 224523 (2011).

[30] L. G. Aslamazov and A. I. Larkin, Zh. Eksp. Teor. Fiz. 68, 766 (1975) [Sov. Phys. JETP 41, 381 (1975)].

[31] I. V. Zolochevskii, Low Temp. Phys. 37, 979 (2011).

[32] S. G. Doettinger, S. Kittelberger, R. P. Huebener, and C. C. Tsuei, Phys. Rev. B 56, 14157 (1997). 